


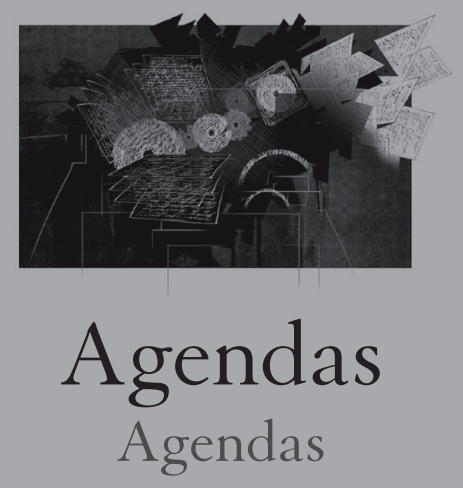

\author{
Marcela Bonnet y Daniela Soledad González \\ La escritura científica en las disciplinas. Análisis del \\ metadiscurso interpersonal en artículos científicos de Química, \\ Biología y Filosofía \\ Scientific Writing in Disciplines: Analysis of the Interpersonal \\ Metadiscourse in Chemistry, Biology, and Philosophy \\ Scientific Articles
}

Teresa Piñeiro-Otero La radio en la investigación comunicativa en España: una línea minoritaria para un medio mayoritario

Radio in Communicative Research in Spain: A Minority Line for a Majority Media 


\section{La escritura científica en las disciplinas: análisis del metadiscurso interpersonal en artículos científicos de química, biología y filosofía}

Scientific Writing in Disciplines: Analysis of the Interpersonal Metadiscourse in Chemistry, Biology, and Philosophy Scientific Articles

A escrita científica nas disciplinas: análise do metadiscurso interpessoal em artigos científicos de química, biologia e filosofia

doi:10.11144/Javeriana.syp35-69.ecda

Recibido: 4 de octubre de 2015

Aceptado: I6 de agosto de 2016 Disponible en línea: I I de noviembre de 2016

Submission Date: Octubre $4^{\text {th }}, 2015$

Acceptance Date: August I $6^{\text {th }}, 2016$ Available Online: November I $^{\text {th }}$, 2016

\section{Origen del artículo}

Este artículo no recibió financiación de ninguna institución ni fue resultado de un proyecto de investigación asociado a una institución.

Marcela Bonnet

Argentina. Licenciada en Lengua y Literatura. Profesora de la Universidad Nacional de Río Cuarto, Instituto de Formación Docente Continua (Villa Mercedes),Conicet. Teléfono 00540358 154176372.marcelabonnet@ hotmal.com

Daniela Soledad González

Argentina. Licenciada en Letras. Profesora de la Universidad Nacional de Cuyo, Conicet. Teléfono 00540261 155612489. gonzalezdanielasoledad@yahoo.com.ar 


\section{Resumen}

Este artículo indaga, a través de un estudio comparativo entre artículos científicos, sobre cómo los autores se construyen en cuanto enunciadores académicos en diversas disciplinas. Se toman las categorías de metafunción interpersonal de Halliday y de metadiscurso de Hyland. A partir de una metodología de análisis cualitativa, se analiza un corpus compuesto por tres series: serie A (artículos de filosofía), B (artículos de biología) y C (artículos de química). Los resultados muestran que los artículos de filosofía presentan mitigadores y enfatizadores de modo homogéneo a lo largo de todo el texto, debido a la intención predominantemente argumentativa que existe en ellos; en cambio, en los artículos de biología y química, se registra una mayor presencia de modalizadores cuando se destaca la importancia del objeto por estudiar o cuando se discuten los resultados. Los artículos de filosofía presentan marcas de primera persona, no hallados en los textos de otras disciplinas.

Palabras clave: conocimiento; disciplinas; escritura científica; discurso interpersonal

\section{Abstract}

This article inquires, by means of a comparative study between scientific articles, about how authors are constructed as academic speaking subjects in different disciplines. We took the categories of Halliday's interpersonal metafunction and Hyland's metadiscourse. Based on a qualitative analysis methodology, we analyze a corpus made of three series: A series (philosophy articles); B series (biology articles) and; C series (chemistry articles). Results show that philosophy articles show mitigating and emphasizing elements homogenously across the whole texts, due to the prevailing argumentative intention existing in them. To the contrary, in biology and chemistry articles there is a greater presence of modifiers when the importance of the object of the study is highlighted or when results are discussed. Philosophy articles show first person markers not found in other disciplines.

Keywords: knowledge; disciplines; scientific writing; interpersonal discourse

\section{Resumo}

Este artigo explora, através de um estudo comparativo entre artigos científicos, como é que os autores são construídos como enunciadores acadêmicos em variadas disciplinas. As categorias metafunção interpessoal de Halliday e metadiscurso de Hyland são tomadas. A partir de uma metodologia de análise qualitativa, analisa-se um corpus composto por três séries: série A (artigos de filosofia), B (artigos de biologia) e C (artigos de química). Os resultados mostram que os artigos de filosofia apresentaram apaziguadores e acentuadores de modo homogêneo ao longo do texto tudo, devido à intenção predominantemente argumentativa que existe neles. Em vez disso, nos artigos de biologia e química há registo de maior presença de modalizadores quando destacada a importância do objeto de estudo, ou quando discutidos os resultados. Os artigos de filosofia apresentam marcas de primeira pessoa não encontradas nos textos de outras disciplinas.

Palavras-chave: conhecimento; disciplinas; escrita científica; discurso interpessoal 
Marcela Bonnet y Daniela Soledad González

\section{La escritura científica en las disciplinas: análisis del metadiscurso interpersonal en artículos científicos de química, biología y filosofía}

La escritura académico-científica en las disciplinas

Las disciplinas científicas no solo constituyen un espacio de construcción de conocimiento, sino también de regulación de formas de comunicación (Sánchez, 2011). En otras palabras, conforman no solo un espacio conceptual, sino también uno discursivo y retórico (Alvarado y Cortés, 2001). Para Sánchez (2011, p. 43), "el texto científico es una construcción lingüística delimitada que funciona en un medio social; es decir, hay una retórica oficial de la ciencia que nos impone una manera de escribir".

Un planteamiento similar lo hallamos en las corrientes de estudio que centran su interés en las formas en que se construye el significado dentro de las disciplinas, como las denominadas lenguaje a través del currículum (LAC) y escritura a través del currículum (WAC). ${ }^{1}$ Estas líneas de investigación consideran que en el discurso de las comunidades disciplinares las prácticas discursivas resultan inescindibles de las prácticas sociales (Markovich y Morán, 1998). ${ }^{2}$ Si tenemos en cuenta la noción de comunidad discursiva, pensamos en el conjunto social de las personas implicadas en la construcción del conocimiento en un área determinada. Este conjunto social incluye no solo a los investigadores que escriben textos científicos, sino también a quienes los evalúan y a quienes los leen (Sánchez, 2011).

Debido a la importancia que posee dicha comunidad en la regulación de los géneros discursivos, existe siempre un grado de tensión entre las exigencias de esta, por un lado, y el autor individual, por el otro, pues se espera que este no solo respete las tradiciones discursivas que la comunidad impone, sino que también adopte un posicionamiento particular frente al conocimiento (Bonnet y Escalarea, 2014). El autor de artículos científicos debe configurar discursivamente una identidad que le posibilite construirse como un sujeto portador de saber, experto en su disciplina, capaz de producir conocimiento científico relevante y original en el campo en el que se desempeña.

Esto implica no solo proporcionar información, sino también producir el efecto deseado en los interlocutores. Por estos motivos, según el lugar que ocupe el autor en la comunidad discursiva que integra y según cuál sea el efecto perlocutivo perseguido en su escrito, recurrirá a determinadas estrategias para proyectarse como persona y seña- 
Marcela Bonnet y Daniela Soledad González | La escritura científica en las disciplinas

lar el grado de certeza del contenido proposicional de su discurso (Beke, 2005).

En este artículo, nos proponemos indagar de manera comparativa sobre el metadiscurso interpersonal en artículos científicos pertenecientes a diversas ciencias, con la finalidad de averiguar si hallamos algún tipo de correlación entre la manera cómo los autores se configuran como enunciadores de artículos científicos y el marco disciplinar en el que escriben estos artículos. La noción de metadiscurso, también conocida como "discurso sobre el discurso" (Hyland, 1998, p. 437), engloba un conjunto de procedimientos empleados para organizar un texto de manera explícita y señalar actitudes del emisor respecto del material y de la audiencia.

En nuestro recorrido bibliográfico, hallamos estudios referidos al metadiscurso en diversos géneros, en distintas lenguas y desde distintas perspectivas. Algunos de ellos son los siguientes: estudios comparativos interculturales de textos académicos argumentativos (Crismore, Markkanen y Steffensen, 1993), análisis de ensayos sobre geografía (Siew Mei, 2013), exploración de abstracts de artículos científicos en lingüística aplicada y economía (Khedri, Swee y Foad, 2013), análisis de textos turísticos (Labarta-Postigo y Suau-Jiménez, 2006), de viaje (Almeida y González, 2012), estudio de la citación en artículos sobre ciencias sociales y humanas (Sánchez, 2015), indagaciones sobre los géneros discursivos académicos y la alfabetización académica en educación superior (Swales, 1990, 1993; Swales y Burke, 2003; Bazerman et al, 2005; Sánchez, 2015), estudios de la modalidad epistémica y evidencialidad en artículos científicos (Ferrari, 2009a, 2009b) y el metadiscurso interpersonal en artículos de investigación correspondientes a textos de pedagogía (Beke, 2005).

Sin embargo, no se han encontrado análisis centrados en disciplinas científicas desde una perspectiva comparativa que tiendan a valorar los modos particulares de construcción del conocimiento científico. Retomamos una versión modificada de la taxonomía de Crismore, Markkanen y Steffensen (1993), que Hyland emplea en la que distingue las dimensiones textual e interpersonal y reconoce categorías específicas en cada una de ellas.

Nos centramos en las categorías vinculadas al metadiscurso interpersonal (modalizadores, marcadores de actitud, marcadores relacionales y marcadores personales), categorías trabajadas 
también por Beke (2005), con la finalidad de analizar a través de estas cómo los autores se posicionan para legitimar su voz en la comunidad discursiva a la que pertenecen. Concretamente, se estudia un corpus de quince artículos de revistas científicas electrónicas, correspondientes a diversas áreas del conocimiento. Se entiende por artículo científico aquella producción original que resulta publicada en una revista de contenido científico, tecnológico o académico luego de una evaluación por pares ciegos, que es producto de procesos de investigación, reflexión o revisión (Sánchez, 2012).

Los artículos de investigación que se analizan son originales (que introducen novedades científicas demostradas), de revisión de problemas ya instalados en el ámbito de la biología y de la química y de reflexión filosófica. La perspectiva teórica de abordaje de los textos es el análisis del discurso desde las categorías de metafunción interpersonal (Halliday, 1994) y metadiscurso interpersonal (Hyland, 1998), con un enfoque comparativo.

La muestra analizada está conformada por tres series: serie A, cinco artículos de filosofía; serie $\mathrm{B}$, cinco artículos de biología; serie $\mathrm{C}$, cinco artículos de química. ${ }^{3}$ Se advirtió que en ellos el metadiscurso empleado por los enunciadores académicos tiene como objetivo principal generar un efecto de verosimilitud, que es propio del lenguaje especializado. Dicho efecto varía según la disciplina de la que se trate, y por ello las estrategias metadiscursivas empleadas también.

A continuación, se desarrollan las nociones de metafunción interpersonal (Halliday, 1994) y metadiscurso interpersonal (Hyland, 1998), que vertebran el análisis de los datos obtenidos, y se comentan los resultados hallados a partir del análisis del corpus.

\section{La noción de metadiscurso interpersonal}

Halliday (1994) plantea que existen tres niveles de significado derivados de las metafunciones del discurso; estos son el textual, el interpersonal y el ideacional (o experiencial). Se trata de tres hebras que conforman un hilo.

El nivel textual contiene unidades de flujo informativo: las emisiones. El nivel interpersonal contiene unidades de interacción, que son, en general, combinaciones de proposiciones y propuestas (si se piensa en un diálogo, por ejemplo). El nivel ideacional hace referencia al flujo de eventos del que se habla, a la construcción de episodios, y sus unidades son las figuras. ${ }^{4}$

Dentro de estos niveles se pueden diferenciar diversos recursos utilizados por el enunciador. Un ejemplo de estos mecanismos, que actúa en el ámbito interpersonal, es la realización de la modalidad epistémica a través de expresiones, como no creo que, que traslada una característica del dictum al ámbito más subjetivo de las creencias del hablante, en lugar del uso de probablemente, por ejemplo.

En el dominio de las órdenes, lo más prototípico es que el emisor se oculte y no se diga explícitamente Te ordeno que... por cortesía. En estos casos, se está ante realizaciones metafóricas de propuestas (proposals) mediante proposiciones. Por ejemplo, proposiciones como Vote against... pueden parafrasearse por propuestas como I urge you to vote against..., razón por la cual Halliday (1994) propone que pueden ser llamadas proposiciones sustantivas, pues cumplen la función de objeto directo de un verbo de decir implícito.

La noción de metafunción interpersonal propuesta por Halliday (1994) es recuperada por Hyland (1998) como metadiscurso interpersonal. Según Hyland (1998), en el uso que los hablantes hacen del metadiscurso, el enunciador muestra al interlocutor de qué manera las distintas partes del discurso se relacionan entre sí, también el uso del metadiscurso interpersonal deja entrever las evaluaciones y las actitudes propias ante aquello de lo que se habla y sobre aquellos a los que se hace hablar en el enunciado. A su vez, mediante recursos metadiscursivos, el enunciador intenta ejercer influencia en la recepción del contenido proposicional de su texto, por ejemplo, a través de los grados de intimidad, distancia e implicación del lector ${ }^{5}$ que el autor establece en su texto.

Como ya mencionamos, entre los principales recursos que postula Hyland (1998), podemos mencionar los modalizadores, los marcadores de 
actitud, los marcadores relacionales y los marcadores personales.

Los modalizadores tienden a atenuar o acentuar el posicionamiento del enunciador respecto de lo enunciado, por lo que podemos hacer una distinción entre mitigadores y enfatizadores. Los primeros indican la decisión que toma el autor de no comprometerse totalmente con la proposición expresada; la información se presenta como una opinión más que como un hecho. Un recurso dentro de esta categoría es el uso del tiempo condicional. Los enfatizadores, por su parte, marcan el grado de compromiso con la información y destacan la fuerza proposicional del discurso.

En segundo lugar, los marcadores de actitud señalan la actitud afectiva del autor, el acuerdo, la importancia, la obligación, etc. Estas actitudes suelen ser señaladas fundamentalmente por adverbios y adjetivos valorativos, verbos de actitud y marcas tipográficas, como subrayados y negritas. En tercer lugar, los marcadores relacionales están en general dirigidos al lector y son una invitación a participar en el discurso, como el uso de la primera persona del plural (plural inclusivo). Por último, los marcadores de persona permiten que los autores presenten sus ideas o perspectivas a través, fundamentalmente, del uso de la primera persona del singular.

\section{Metodología y corpus}

El corpus revisado está conformado por tres series de cinco artículos cada una, según las disciplinas a las que pertenecen los artículos: serie $\mathrm{A}$, artículos de filosofía; serie $\mathrm{B}$, artículos de biología; y serie C, artículos de química, extraídos de revistas científicas electrónicas. El criterio con el que se seleccionó la muestra fue el de conformar un corpus que posibilite comparar los recursos metadiscursivos empleados por los autores en la escritura de ciencias diversas: sociales y humanas (en la revista de filosofía), ciencias exactas (en la revista de química) y ciencias naturales (en la de biología).

Partiendo del supuesto de que "los recursos modales léxicos y léxico-gramaticales difieren cualitativa y cuantitativamente según las distintas disciplinas" (Ferrari, 2009b, p. 10), realizamos un análisis cualitativo de tipo comparativo, en el que ejemplificamos el funcionamiento de los recursos metadiscursivos empleados y buscamos describir y contrastar su forma de utilización.

Los recursos metadiscusivos focalizados son los desarrollados en el apartado anterior, en especial los modalizadores llamados atenuadores, que se relacionan con los adverbios de modalidad dubitativa y los enfatizadores, relacionados con los adverbios epistémicos y evidenciales, que actúan como reforzadores del valor de verdad.

\section{Análisis del corpus}

La presencia de mitigadores y enfatizadores y de algunos marcadores de actitud pudo observarse en las tres series estudiadas. No obstante, se advierten diferencias significativas entre la serie A (artículos de filosofía) y las series B y C (artículos de biología y química). Mientras en la serie A la presencia de modalizadores se halla indistintamente a lo largo de todo el texto, en las series $\mathrm{B}$ y $\mathrm{C}$ se registró mayor presencia de estos o bien cuando se destacaba la importancia del objeto por estudiar o bien cuando se discutían los resultados de los análisis realizados.

Dicha diferencia parece estar relacionada con las secuencias ${ }^{6}$ propias y predominantes que se ponen en juego en los textos de las diversas disciplinas. Mientras que en los artículos de filosofía la información se estructura bajo formas predominantemente argumentativas, en los textos de biología y química advertimos que conviven fundamentalmente las secuencias descriptivas y explicativas. En estos dos últimos tipos de textos, los modalizadores (mitigadores y enfatizadores) aparecen en algunas secuencias descriptivas que tienden a destacar la importancia del objeto por estudiar, así como en secuencias explicativas que presentan resultados y discusiones.

Los fragmentos (1) y (2) provenientes de artículos de biología — extraídos de los apartados "Discusión” y “Conclusiones", respectivamenteconstituyen secuencias de tipos dominantemente explicativas, en las que, por un lado, se destaca la importancia de los resultados obtenidos a través 
de marcadores de actitud, Es importante notar que, y al mismo tiempo se utilizan modalizadores mitigadores que enuncian las conclusiones a las que el autor llega como una probabilidad más que como una certeza. Esto puede advertirse a través del uso de mitigadores, como podrían o pueden: ${ }^{7}$

(1) Es importante notar que en ambos trabajos la mayor densidad de venados fue encontrada en sitios con bosques de coníferas, que se ubican a mayores altitudes. Tanto en el trabajo de Ortiz-Martínez (2005), como en este trabajo, se reportaron disturbios antropogénicos derivados del manejo forestal. En particular, durante la realización de este trabajo se llevaron a cabo actividades de saneamiento forestal en la zona, que derivaron en la presencia constante de trabajadores, introducción de maquinaria y camiones de carga. Asimismo, Ortiz-Martínez et al. (2005) reportan perturbaciones ocasionadas por el tránsito de vehículos y trabajadores, así como ruido producido por el aprovechamiento forestal. Estos factores podrían afectar la presencia, así como las preferencias de hábitat de los venados en la zona, lo que puede ocasionar cambios en la distribución de los venados, obligándolos a refugiarse en los sitios más altos e inaccesibles (Leal, Canales, Aranis y Gonzáles, 2011, p. 115).

En el caso de (2) se observan los mitigadores probablemente, permiten sugerir, tendría:

(2) Por otra parte, los mayores valores de MO - materia orgánica- en el área de estudio se observaron en los sectores T2 y T3, lo cual probablemente se asocie a su ubicación en el área de influencia urbana en la bahía y a la actividad de desembarque de la actividad pesquera local. Estos resultados permiten sugerir que la zona urbana localizada frente a T3 tendría una mayor injerencia sobre la composición biogénica de los sedimentos de fondo de la bahía (Moraga, Galán, Rosello Mora, Araya y Valdés, 2014, p. 240).

También se hallaron otros marcadores de actitud en fragmentos explicativos. Aparecen, principalmente, al comienzo del artículo, y su función es la de introducir una dimensión afectiva relacionada con la información expuesta. Estos marcadores tienden a utilizarse cuando los enunciadores deben destacar la importancia del tema por investigar. En (3) pueden observarse marcas como desafortunadamente y está bien establecido:

(3) Desde su descubrimiento, los plaguicidas químicos han sido el método más empleado para el control de mosquitos transmisores de enfermedades. La aplicación de insecticidas en fase adulta, tanto en tratamientos intra como extra domiciliarios se utiliza para obtener una reducción rápida de las poblaciones en los casos de epidemia trasmitidas por vectores. Desafortunadamente, dicha reducción suele ser transitoria si no se aplican correctamente los plaguicidas. Está bien establecido que la resistencia a los mismos puede desarrollarse rápido en algunas poblaciones y lentamente en otras (Leyva, Marquetti, French, Montada, Tiomno y Tacoronte, 2013, pp. 75-76).

Por último, otras marcas de actitud encontradas son, por ejemplo, por excelencia, gran importancia, importante y fundamental, que pueden apreciarse en el fragmento (4):

(4) El venado cola blanca es la especie usada por excelencia para actividades cinegéticas, por lo que tiene una gran importancia ecológica como herbívoro y presa, formando parte de redes tróficas, comunidades y ecosistemas diversos. Es un importante regulador de poblaciones vegetales [...] Actúa como un factor limitante fundamental para poblaciones de grandes predadores (Piña, 2014, p. 115).

A diferencia de lo observado en los artículos de biología y química, se advirtió que en los artículos de filosofía los marcadores de actitud y modalizadores se hallan a lo largo de toda la materialidad textual. En especial, cuando el enunciador tiene la necesidad de exponer sus argumentos, como puede advertirse en los fragmentos (5), (6), (7) y (8):

(5) No hace falta entrar en la discusión de si 
para Tomás de Aquino el Dios de Aristóteles (o incluso el de Platón) crea o no crea para afirmar que unas de las principales luchas filosóficas sostenida por parte de los pensadores cristianos del siglo XIII fue el intento de clarificar más y más el hecho de que Dios era una creador libre (Argüello, 2011, p. 28).

(6) Es decir, sea cierto o no que como sostiene Pegis, los maestros medievales cristianos pensaron que los griegos y los árabes no habían enseñado la creación, de lo que no se puede dudar jamás es que esos maestros estaban empeñados en dar explicaciones mucho más hondas y ricas que las precedentes al respecto (Argüello, 2011, p. 30).

(7) La filosofía de la ciencia elaborada por G. Deleuze puede ser remitida a una variante muy precisa de la epistemología francesa en tanto deriva, en lo esencial, de aquella tendencia ontológica abierta por $\mathrm{H}$. Bergson que sostiene la independencia de la filosofía respecto de lo científico y orienta el pensamiento filosófico de lo científico no tanto hacia la investigación de las condiciones formales o históricas de su conocimiento como hacia la elaboración de un concepto capaz de pensar aquello que la ciencia es. Tan profunda resulta esta filiación que la casi totalidad de filosofía de la ciencia deleuziana puede ser entendida como organizada en torno a una problemática de índole ontológica: ¿cómo concebir esa ciencia que se dispone a la altura de un ser que es repetición de la diferencia y un pensar que sólo se ejerce en tanto que diferencia en la repetición? Sea como fuere, la consideración del proyecto ontológico que condiciona la aproximación epistemológica deleuziana, no cesa de despertar la mayor de las confusiones (Gallego, 2011, p. 93).

(8) Antes de introducir la discusión suscitada por estas tesis habermasianas, tenemos que pasar por el rodeo de una advertencia: la que nos hace, entre otros, Amy Allen en un artículo que nos invita, desde el título, a una "re-consideración" del "debate Foucault-Habermas", pero inmediatamente nos sorprende diciendo que "El debate Foucault-Habermas nunca existió [was a nonevent]. Las razones para ello fueron tanto personales - la muerte prematura de Foucault - como filosóficas — la aparente incapacidad de Foucault y Habermas para ponerse de acuerdo en un tema para debatir. Sean cuales fueren las razones, ningún intercambio formal de ideas entre Foucault y Habermas ocurrió jamás; [...] lo que se conoce como el debate Foucault-Habermas es principiante un producto de la literatura secundaria sobre estos dos pensadores (Cormick, 2011, pp. 63-64.)

Respecto de los marcadores relacionales y de persona se halló una diferencia sustancial entre los artículos de biología y química por un lado, y los de filosofía, por el otro. En el caso de los primeros, se encontró una abundante cantidad de nominalizaciones tendientes a esconder la acción del sujeto agente, estrategia de reificación propia de la rigurosidad que suelen buscar este tipo de disciplinas. Este dato es significativo, porque habla de una ausencia del recurso metadiscursivo de la marcación de persona. A continuación, se presentan ejemplos, como botón de muestra:

(9) El análisis reveló un elevado porcentaje de conversión de la mezcla monoterpénica mayoritaria primaria del aceite de trementina $(\alpha / \beta$-pinenos, $>70 \%$ ), por el mecanismo carbocation- $\alpha$ oxidación, en sistemas monocíclicos oxigenados tipo terpinolenos y limonenos, y derivados pulegona y verbenona (Leyva, 2013, p. 114).

(10) A partir del estudio de material depositado en colecciones de referencia de entomología, y de material colectado en sectores aledaños a la ciudad de Leticia, Amazonas, se realiza la re-descripción de la especie, analizando las variaciones en el patrón de pigmentación de machos y hembras y aportando información sobre su biología e historia natural. ("Sobre la taxonomía y biología de cyclocephala mannheimsi endrödi, 1964 (Gasca Álvarez, 2014, p. 174).

Esta estrategia de encapsulamiento de hipótesis o afirmaciones en etiquetas nominales es un recurso muy utilizado, fundamentalmente, en los 
artículos propios de las ciencias naturales, como sostienen Halliday y Martin (1993, p. 15): ${ }^{8}$

El desarrollo de la jerga técnica y el pensamiento científico es, en realidad, un fenómeno que tiene una base gramatical: por ejemplo, la oración "el aerogel fue posteriormente desarrollado por..." se transforma en la nominalización "el posterior desarrollo del aerogel", que permite su utilización como elemento en una nueva oración, más compleja conceptualmente, y que colabora así con la construcción de una teoría científica.

También observamos algunas estrategias de agentivación mediante las cuales la acción del sujeto es realizada por un agente inanimado (el estudio, el análisis, etc.). El sujeto enunciador tiende a presentarse atenuado a través de estas formas como una manera de otorgar rigurosidad científica al dictum:

(11) El estudio consideró solamente a las hembras de Sprattus fueguensis, dado que los cambios más notables y fáciles de percibir en la actividad reproductiva de los peces ocurre a nivel de los ovarios (Leal et al., 2011, p. 45).

(12) El análisis de la actividad reproductiva en relación a la longitud consideró un total de 546 hembras muestreadas (Leal et al., 2011, p. 45).

También es abundante, en este tipo de textos, el uso del se impersonal para hacer referencia a las acciones realizadas por los autores, como se muestra en (13), (14) y (15):

(13) Se llevó a cabo la transformación catalítica del limoneno usando cuatro materiales basados en molibdeno y cobalto (Salamanca, Licea, Echavarría, Farol y Palacio, 2009, p. 93).

(14) La sardina austral, Sprattus fuegensis, ha sido recientemente identificada en la captura de peces pelágicos en el mar interior de Chiloé. A pesar de su creciente importancia económica aún se desconocen aspectos básicos de su biología reproductiva. Se describe la actividad reproductiva de las hembras de esta especie en base a la variación mensual del índice Gonadosomático (IGS) [...] se estudia además el proceso de maduración en la longitud corporal de las hembras durante la época del desove [...]. Se discute que el aumento de la actividad reproductiva en primavera, así como la maduración a mayor tamaño corporal, podría ser parte de la estrategia reproductiva de la especie (Leal et al., 2011, p. 46).

(15) Se evaluó la densidad poblacional y se caracterizó el hábitat del venado de cola blanca (Piña, 2014, p. 114).

Estas estrategias de ocultamiento del sujeto fueron recurrentes en los artículos de biología y de química, mientras que en los artículos de filosofía se halló una fuerte presencia de la primera persona del plural (referida al autor), como lo muestra el siguiente ejemplo:

(16) Nuestro aparato de lectura procurará vislumbrar algunos inconvenientes de la perspectiva filosófica y estética del autor (Di Paola, 2011, p. 80).

Estos marcadores de persona no aparecieron en los artículos de biología y de química consultados. Mediante esta forma el enunciador se hace responsable de su discurso y, por otra parte, evidencia una carga de subjetividad más acentuada que la que puede observarse en los artículos propios de las ciencias naturales. En (17) se provee otro ejemplo de este tipo de recursos, esta vez con la primera persona del singular.

(17) El tema general de mi artículo refiere a lo que Axel Honneth denomina "sentimientos negativos" o de agravio moral. Mi interés es dilucidar cómo estos sentimientos motivan diferentes tipos de reacción grupal. [...] así mismo, buscaré precisar si para Honneth puede afirmarse de que exista manipulación de los sentimientos (Abril, 2011, p. 13). 


\section{Conclusiones}

En este trabajo, se indagó sobre el uso de recursos metadiscursivos interpersonales en el ámbito académico-científico en disciplinas diversas, como la filosofía, la biología y la química, desde un enfoque comparativo. A pesar de reconocer la existencia de gran cantidad de investigaciones sobre el metadiscurso centradas en el discurso académico (Hyland, 1998; Swales, 1990, 1993; Swales y Burke, 2003; Bazerman et al., 2005; Sánchez, 2015; Siew Mei, 2013), no hallamos estudios que muestren comparativamente cómo los autores de artículos científicos construyen de diversas maneras su identidad discursiva según su comunidad de pertenencia.

El artículo se centró en las categorías de metafunción interpersonal (Halliday, 1994) y metadiscurso interpersonal (Hyland, 1998). Para ello, retomamos una taxonomía modificada de Crismore, Markkanen y Steffensen (1993), que Hyland emplea, a saber: modalizadores, marcadores de actitud, marcadores relacionales y marcadores personales. En los artículos científicos trabajados, es posible hallar variados recursos lingüísticos de los que se valen los enunciadores para apropiarse e inscribir el conocimiento en un determinado campo disciplinar, atendiendo a diversos factores interpersonales.

Pudimos observar que los modalizadores (mitigadores y enfatizadores) y algunos marcadores de actitud se hallan presentes en las tres series estudiadas. No obstante, se advirtieron diferencias significativas entre la serie A (artículos de filosofía) y las series B y C (artículos de biología y química). Mientras en la serie A la presencia de modalizadores y enfatizadores se observó indistintamente a lo largo de todo el texto, debido a la intención predominantemente argumentativa que existe en estos artículos, en las series B y C se registró una presencia de estos, bien cuando se destaca la importancia del objeto por estudiar, bien cuando se discuten los resultados de los análisis realizados. En otras palabras, los modalizadores parecen concentrarse en partes específicas cuando se trata de los artículos pertenecientes a las ciencias exactas y naturales.
Respecto de los marcadores relacionales, estos se manifiestan en niveles que van desde el máximo grado de ocultamiento del sujeto enunciador (nominalizaciones), pasando por la impersonalidad (estas dos características se advierten fundamentalmente en los artículos de biología y química), hasta llegar a la explicitación del sujeto a partir de marcas de primera persona. Este último rasgo solo se halló en los artículos de filosofía.

En tal sentido, no podemos dejar de mencionar que en su estudio acerca de la metafunción interpersonal en artículos científicos pertenecientes al área de educación, Beke (2005) advierte que los marcadores relacionales y actitudinales son los más empleados por los investigadores de la educación. Es decir, en estos artículos de ciencias sociales y humanas, al igual que en los de filosofía que hemos analizado en esta instancia, hay una tendencia de los autores a preocuparse por proyectarse como persona, preocupación que parece no ser compartida por los autores de artículos ligados a las ciencias exactas y naturales, quienes prefieren formas desagentivadas en las que más bien tienden a ocultar al sujeto de la enunciación.

Cabe aclarar que esta investigación tiene el alcance de un estudio descriptivo sobre un corpus relativamente acotado, pero resulta extensible a través de estudios estadísticos de corpus, con implicancias mayores para los estudios del metadiscurso y el discurso académico-científico.

Con esta indagación, se pretendió establecer que la construcción del objeto de conocimiento científico en el lenguaje especializado de las ciencias está íntimamente relacionada con usos metadiscursivos de los que se valen los enunciadores para agregar a la información proposicional consideraciones propias (en algunos casos sin resaltar su subjetividad, en otros acentuándola), y persuadir a sus lectores sobre la plausibilidad de sus ideas.

\section{Referencias}

Adam, J. (1992). Les textes: types et prototypes: Récit, description, argumentation, explication et dialogue. París: Nathan. 
Almeida, F. y González Cruz, M. (2012). Exploring male and female voices through epistemic modality and evidentiality in some modern english travel texts on the canaries. Research in Language, 3(10), 323-343.

Alvarado, M. y Cortés, M. (2001). La escritura en la universidad: repetir o transformar. Lulú Coquette: Revista de Didáctica de la Lengua y la Literatura, 1(1).

Bazerman, C., Little, J., Bethel, L., Chavkin, D. y Garufis, J. (2005). Reference guide to writing across the curriculum. Indiana: Parlor Press.

Beke, R. (2005). El metadiscurso interpersonal en artículos de investigación. Signos, 38(57), 7-18.

Bonnet, M. y Escalarea, L. (2014). Identidades discursivas en la escritura de las disciplinas: una enseñanza posible, una responsabilidad compartida. En P. Rosales y M. ${ }^{\text {a del C. Novo }}$ (comps.), Lectura y escritura en carreras de ciencias humanas y sociales: ideas y experiencias de enseñanza. Buenos Aires: Noveduc.

Cinto, M. (2009). La nominalización: obstáculo para la comprensión lectora. Educación, Lenguaje y Sociedad, 6(6), 171-186.

Crismore, A., Markkanen, R. y Steffensen, M. (1993). Metadiscourse in persuasive writing: A study of texts written by american and finnish university students. Written Communication, 10, 39-71.

Downing, A. (1991). La metáfora gramatical de M. A. K. Halliday y su motivación funcional en el texto. RSEL, 21(1), 109-123.

Ferrari, L. (2009a). Modalidad epistémica y evidencialidad en las conclusiones de artículos de investigación. Debate Terminológico, 6.

Ferrari, L. (2009b). Marcadores de modalidad epistémica y evidencial en el análisis de las conclusiones de artículos de disciplinas distintas. ALED: Revista Latinoamericana de Estudios del Discurso, 2(9), 5-24.

Halliday, M. (1994). An introduction to functional grammar (2. ${ }^{\text {a }}$ ed.). Londres: Edward Arnold.

Hyland, K. (1998). Persuasion and context: The Pragmatics of Academics Metadiscourse. Journal of Pragmatics, 4(30), 437-455.
Khedri, M., Swee Heng, C. y Foad Ebrahimi, C. (2013). An exploration of interactive metadiscourse markers in academic research article abstracts in two disciplines. Discourse Studies, 15, 319-331.

Labarta-Postigo, M. y Suau-Jiménez, F. (2006). Análisis del metadiscurso en textos especializados turísticos: los matizadores discursivos y la pronominalización en alemán y español. Ponencia presentada en Congreso ALED, Valparaíso (Chile).

Markovich Ravena, J. y Morán Ramírez, P. (1998). La escritura a través del curriculum. Signos, 31(43-44), 165-171.

Mondada, L. (1995). La construction discursive des objets de savoir dans l'écriture de la science. Réseaux, 71(13), 55-77.

Müller, G. (2007a). Adverbios de modalidad epistémica y evidencialidad en artículos de investigación y conferencias académicas: funciones metadiscursivas y aspectos cognitivos. Romanistischen Jahrbuch, 58, 329-364.

Müller, G. (2007b). Metadiscurso y perspectiva: funciones metadiscursivas de los modificadores de modalidad introducidos por como en el discurso científico. Revista Signos, 40 (64), 357-387.

Navarro, F. y Revel Chion, A. (2013). Escribir para aprender: disciplinas y escritura en la escuela secundaria. Buenos Aires: Paidós.

Ricoeur, P. (1980 [1975]). La metáfora viva (trad. Agustín Neira). Madrid: Ediciones Europa.

Siew Mei, W. (2013). Certainty judgements and the status of propositions in undergraduate essays. RELC Journal, 44, 279-302.

Sánchez Upegui, A. (2011). Manual de redacción académica e investigativa: cómo escribir, evaluar y publicar artículos. Medellín: Fundación Universitaria Católica del Norte.

Sánchez Upegui, A. (2012). Análisis lingüístico de artículos de investigación en ciencias sociales y humanas. Lingüistica y Literatura, 67(2), 105-121.

Sánchez Upegui, A. (2015). Análisis lingüístico de la citación en artículos de ciencias sociales y humanas. Revista Lasallista de Investigación, 12(1), 101-126. 
Swales, J. (1990). Genre analysis: english in academic and research settings. Cambridge: Cambridge University Press.

Swales, J. (1993). Genre and engagement. Revue belge de philologie et d' histoire, 71(3), 687-698.

\section{Apéndices}

\section{Corpus analizado}

\section{Artículos de filosofía}

Abril, F. (2011). Sentimientos negativos y dominación social. Philosophia, 72, 79-92.

Argüello, S. (2011). Creación y libertad: la cuestión tras la discusión entre Pegis y Lovejoy. Philosophia, 72, 25-35.

Cormick, C. (2011). Situacionalidad del conocimiento, "racionalismo" y dimensión "externa" de la verdad: una intervención en el (no-)debate Habermas-Foucault. Philosophia, 71, 59-78.

Di Paola, E. (2011). Ontologías y estéticas postrepresentación: problemas de filosofía del arte de Arthur Danto, de Esteban Di Paola. Philosophia, 71, 79-92.

Gallego, F. (2011). El concepto deleuziano de "functor". Philosophia, 71, 93-110.

\section{Artículos de biología}

Díaz, P. y Campos, B. (2014). Ontogenia de la concha larval y postlarval de cuatro especies de bivalvos de la costa del Pacífico sureste. Revista de Biología Marina y Oceanografía, 2(49), 175-191.

Leal, E., Canales, M., Aranis, A. y Gonzáles, M. (2011). Actividad reproductiva y longitud de madurez de sardina austral Spattus fueguensis en el mar interior de Chiloé, Chile. Revista de Biología Marina y Oceanografía, 1(46), 43-51.

Leyva, M., Marquetti, M., French, L., Montada, D., Tiomno, O. y Tacoronte, J. (2013). Efecto de un aceite de trementina obtenido de Pinus tropicalis Morelet sobre la biología de una cepa de Aedes (Stegomyia) aegpti, en Linnaeus, resistente a insecticidas. Anales de Biología, 35, 75-84.

Moraga, R., Galán, A., Rosello Mora, R., Araya, R. y Valdés, J. (2014). Composición de la comunidad
Swales, J. y Burke, A. (2003). It's really fascinating work: Differences in evaluative adjectives across academic registers. Language and Computers, 46(1), 1-18.

procariota involucrada en la producción de nitrógeno en sedimentos de la bahía Mejillones. Revista de Biología Marina y Oceanografía, 49(2), 225-241.

Piña, E. (2014). Densidad poblacional y caracterización de hábitat del venado cola blanca en un bosque templado de Oaxaca, México. Acta Zoológica Mexicana, 1(30), 114-134.

\section{Artículos de química}

Buitrago, E., Calderón, L., León, A., Brunetto, R. y Gallignani, M. (2010). Desarrollo y validación de un método espectrofluorométrico para la determinación de la furosemida en formas farmacéuticas sólidas. Avances de Química, 1(5), 15-25.

Castillo, D., Lanza, J. y Crescente, O. (2010). Identificación preliminar de algunos constituyentes del tallo de Paullinia fuscescens (Sapindaceae) y actividad biológica. Avances de Química, 1(5), 57-61.

Halliday, M. A. K. y Martin, J. R. (1993). Writing science: Literacy and discursive power. Pittsburgh: University of Pittsburgh Press.

Ramírez, C., Olarte, E., Téllez, E. y Palma, M. (2013). Validación del método analítico para la determinación de mercurio total en sangre humana por espectrofotometría de absorción atómica Zeeman RA-915+ con el módulo de pirolisis PYRO-915. Revista Colombiana de Química, 3(42), 326-343.

Salamanca, M., Licea, Y., Echavarría, A., Farol, A. y Palacio, L. (2009). Uso de materiales tipo cobalto-molibdato como potenciales catali- 
zadores en la transformación del limoneno. Avances en Química, 4(3), 93-100.

Valenzuela, L., Ortiz, B. y Pérez, C. (2013). Estudio comparativo del efecto metabólico de

\section{Notas}

1. Este planteamiento surge a fines de la decada de 1960 en el Reino Unido como "lenguaje a través del currículum" (LAC), en respuesta a la crisis de alfabetización. Posteriormente, se acuña el término "escritura a través del currículum" (WAC) con el proyecto de desarrollo de la habilidad de escritura del Instituto de Educación de la Universidad de Londres, a cargo de James Britton y sus colaboradores. El movimiento WAC pasa del Reino Unido a los Estados Unidos a mediados de la década de 1970. Se trata de rescatar, por una parte, las muchas maneras de construir significado, la relación de cada disciplina con el lenguaje; por otra parte, se intenta lograr la convergencia de las distintas áreas hacia un objetivo común: expresarse por escrito eficientemente como un modo de aprendizaje. Para mayores detalles sobre este asunto, consultar Markovich y Morán (1998).

2. La construcción discursiva del conocimiento es analizada también desde disciplinas, como la sociología de las ciencias y el análisis del discurso (Mondada, 1995).

3. Los artículos de la serie A fueron todos extraídos de la revista Philosophia, perteneciente a la Facultad de Filosofía y Letras de la Universidad Nacional de Cuyo. Los de la serie $B$, artículos de biología, fueron extraídos de revistas diversas: Revista de Biología Marina y Oceonografía, Anales de Biología y Acta Zoológica Mexicana. Los artículos que constituyen la serie $C$ están inscriptos en el área de la química y pertenecen a la revista electrónica Avances en Química.

4. La noción de figura es explicada por Ricoeur (1980, p. 206) de la siguiente manera: "Las figuras hacen que el discurso se pueda describir haciéndolo aparecer bajo formas discernibles [...] la figura es la que hace perceptible el discurso". Ricoeur (1980, p. 81) arepas enriquecidas con pectina extraída de guayaba (Psidium guajava L.) o pectina cítrica comercial. Revista Colombiana de Química, 3(42), 302-325.

trae a colación la definición de figura que da Fontanier (1830): "Son las formas, los rasgos o los giros más o menos notables y de un efecto más o menos feliz, por los que el discurso, en la expresión de las ideas, de los pensamientos o de los sentimientos, se aleja más o menos de la posible expresión sencilla y común".

5. Para un análisis más exhaustivo del metadiscurso interpersonal, cf. Müller (2007a, 2007b). La autora propone establecer una correlación entre diferentes subcategorías del metadiscurso interpersonal y adverbios periféricos, como entre recursos metadiscursivos "atenuadores" y adverbios de modalidad dubitativa, o entre recursos "enfatizadores" y adverbios reforzadores del valor de verdad, tanto epistémicos como evidenciales. Parte de la idea de que los estudios sobre los adverbios de modalidad epistémico-evidencial han identificado en estos un valor reforzador o enfático, pero no han desmenuzado esta función general de los adverbios en "funciones metadiscursivas específicas" (Müller, 2007a, p. 329). En eso consiste su trabajo.

6. Adoptamos la noción de secuencia que postula Adam (1992). Desde esta perspectiva, las secuencias se definen por los modos prototípicos de combinación de proposiciones, es decir, tipos relativamente estables de combinación de enunciados, dotados de una organización reconocible por su estructura jerárquica interna (esquema) y por su unidad compositiva (plan). A partir de la noción de secuencia, Adam (1992) sostiene que existen diferentes planos de la organización textual y redefine el texto como una estructura compuesta por secuencias.

7. Las palabras destacadas en negrita pertenecen a las autoras del presente trabajo.

8. Citados en Navarro y Revel (2013, p. 50).

Cómo citar este artículo

Bonet, M. y Soledad-González, D. (2016). La escritura científica en las disciplinas: análisis del metadiscurso interpersonal en artículos científicos de química, biología y filosofía. Signo y Pensamiento, 35(69), 16-28. http://dx.doi.org/10.11144/

Javeriana.syp35-69.ecda 
